\title{
ALARS - Automated Launch And Recovery System for AUVs
}

\author{
Vítor H. Pinto*†, Nuno A. Cruz ${ }^{* \dagger}$, Rui M. Almeida ${ }^{\dagger}$, Carlos F. Gonçalves ${ }^{\dagger}$ \\ ${ }^{*}$ Faculty of Engineering, University of Porto, Portugal \\ $\dagger$ INESC TEC, Porto, Portugal \\ vitorpinto@fe.up.pt,nacruz@fe.up.pt,rui.m.almeida@inesctec.pt, carlos.f.goncalves@inesctec.pt
}

\begin{abstract}
Underwater sensing and mapping operations using autonomous vehicles are becoming widely used. This article describes an automated system to launch and recover an AUV. It can operate in any host platform and can transport any torpedo-shaped vehicle with 0.2 meters of diameter, length up to 3 meters and weight up to $1000 \mathrm{~N}$. The system ensures a restrained transportation of the vehicle and guarantees that it performs a smooth entrance in the water. It was instrumented for continuous status remote monitoring, using linear and angular motion sensors, as well as enables to remotely take control over the operation. Experimental results carried out within the XPRIZE competition demonstration scope are presented.

Keywords-Autonomous Underwater Vehicles, Automated Launching and Recovering, Autonomous Surface Vehicles.
\end{abstract}

\section{INTRODUCTION}

The use of Autonomous Underwater Vehicles (AUVs) has become relatively widespread in research and military applications. However, even with the increasing autonomy of batteries, the limited amount of power carried on-board dictate that these vehicles are not able of performing large transits to the targeted operational area. Usually, these have to be transported and launched on or near the operations scenario. This typically involves a support vessel, with her respective crew members, along with specialized operators responsible for deploying and retrieving the AUV.

Ideally, if the referred tasks were performed autonomously, the reduced operational costs and risk to the crew members lives would be a major improvement, comparing to manually perform them, or to the already used physical auxiliary tools, like winches or cranes.

This work describes an Automated Launch And Recovery System (ALARS) for AUVs. The system is designed to be modular enough, so it can be useful in any operation of this kind.

To clarify, our definition of an automated system refers to performing tasks without direct human intervention. Naturally, this does not prevent that remote control may occur, in the event of unforeseen circumstances, or if the conditions do not allow to perform a safe autonomous operation.

Another further clarification concerns the definition of system boundaries. Thus, it was assumed that the vehicle is mechanically attached for the launch and recovery procedures. After this, it can independently carry out both operations without any external intervention.
A real example is the application of this system by the PISCES team in the Shell Ocean Discovery XPRIZE [1]. For this competition the team's strategy consists in transporting a deep water AUV using an Autonomous Surface Vehicle (ASV) to the defined location, then being launched to perform a sea floor mapping mission.

The paper is organized as following: section I introduces the problem and motivation, section II defines the related work, while in section III the design considerations for the proposed system are specified. In section IV, the mechanical and electrical designs are presented and throughout section $\mathrm{V}$ the software actions and procedures are specified. Further, in section VI the experimental results are presented and finally, section VII will focus the conclusions and future work.

\section{RELATED WORK}

In recent years, the use of multiple cooperative vehicles has grown in response to the uprising challenges of oceans exploration. Teams of multiple heterogeneous vehicles working together have been used, where key features from those vehicles have been exploited to exceed the limits that each vehicle had individually. To increase the autonomy of survey systems in areas too far for AUVs to transit, these need to be paired with other classes of more suitable vehicles [1]. As an example, one class capable of performing considerable large transits are ASVs since, for these, autonomy is a lesser problem. In fact, this type of vehicles can easily transport large amounts of batteries, as well as powerful thrusters that allow them to cover larger areas with a substantial load.

A successful example is the Sentry AUV [2], which has spent 200 days per year in sea operation, transported by a ship. This vehicle is transported by a ship to the mission location, being then launched and recovered with the aid of a crane located on the ship, as well as cables maneuvered by human operators. Authors of paper [2] redirects to a video that shows how these operations are performed [3].

MBARI AUV [4] is also another successful example of an autonomous vehicle that is launched and recovered in an automated way, using a crane placed on the transportation ship. These examples illustrate processes which resort to automated systems requiring, however, human intervention throughout the whole procedure.

Hayashi et al. [5] from International Submarine Engineering Ltd. (ISE), in partnership with Hawboldt Industries Ltd., have 
designed and developed a LARS for their AUV. This system consists in a ramp on top of a ship, that requires nothing more than deck space and power from it. To launch the vehicle there is a winch that makes the vehicle slide offboard and, for recovery, the AUV needs to be remotely driven to the ramp. The ramp needs 2 people to operate it and another person to drive the AUV. The structure has a nose ring that has the winch cable in the middle of it.

Sarda and Dhanak [6] have designed an ASV-based LARS that consists on autonomously recovering an AUV resorting to winches, lateral guides, electromagnets, among other components. The authors use a cable that has a depressor wing structure at its tip to ensure that the cable is always close to the vertical angle. The underwater vehicle aligns with the ASV and with the suspended cable, latching the guides when aligned. Resorting to the 3 available winches and assuming that the AUV has a way of mechanically connect with the surface vehicle, the underwater vehicle is recovered on board.

Also Piskura et al. [7] have developed a system consisting of a robust line, a transponder and a capture nose. The AUV uses Digital Ultra-Short Baseline (D-USBL) for navigation to and homing to the cable. When it arrives there, the capture nose holds to it and the cable pulls the vehicle on top of a structure designed to receive and transport it. This is the one that supports the vehicle during transportation and launches it at the mission site.

\section{DESIGN CONSIDERATIONS}

First, it was necessary to specify the desired system characteristics. It was decided to develop a system capable of, in an automated way, launching and recovering torpedo-shaped bodies with a maximum length of 3 meters and weight of 1000 $\mathrm{N}$, from an undefined host platform. This way, the developed ALARS will be modular enough to be useful for a wide number of situations. The vehicles supposed to be traveling in this system, for example MARES AUV [8], have $0.2 \mathrm{~m}$ of diameter, so it represents a restriction. However, system must be designed to minimize its impact.

Taking into account all the possible uses for a system like this one, but given that the most immediate application will be to participate in the Shell Ocean Discovery XPRIZE competition, some additional requirements were defined:

- Capability of recovering a drifting vehicle, without its intervention - After completing a mission, the AUV can, in some conditions, return to the surface with little or no power on board, since it is built to be naturally buoyant. In that sense it should be possible for the system to actively recover a drifting AUV;

- Waterproof construction - Since the work environment will be the marine one, both electronics and structure should be waterproof. Given that the system is not designed to be immersed, but there is only the possibility of being splashed with water, the protection rating must be, at least, IP66;

- Enable both automated and remote control - As mentioned before, marine operations may, due to the incon- stant environmental conditions, cause a high degree of disturbance to any system. For this reason, the possibility of remote control becomes important, allowing total control of the system and of all its actions, global and individual;

- Independent from the host platform and the vehicle Taking into account that the system must be useful for multiple functions, it should be considered its use for different vehicles and host platforms. Thus, system modularity becomes relevant, making it independent from both the vehicle and host;

- Possibility of movement restriction - without knowledge of sea conditions and, in order to maintain the AUV's integrity, it should be kept restrained during transit. So, the system must be equipped with an immobilization system that keeps the vehicle in place until the launch procedure, at which time it should release it;

- Minimize changes on the AUV - Since any change on the AUV design will have effects on its hydrodynamic, these changes must be minimized.

\section{System Design}

Considering the previously defined requirements, the developed system can be divided into four interconnected areas that will be presented along this section: mechanical structure, consisting of the system skeleton which deals only with the materials and mechanical mounting of the body itself; actuation, that provides the system with some movement ability; sensing, responsible for acquiring state information during the whole operation; gateway box, that joins all the electronic components and act as an input for controlling the system, requiring that the receiving platform only provides a power source and a network connection.

\section{A. Mechanical Structure}

The mechanical structure is built from aluminum profiles $(30 \times 30 \mathrm{~mm})$ with a moving cradle, thus creating a sliding/rising platform which supports and moves the load. This structure weights around $400 \mathrm{~N}$ and occupies a volume of 2.60 x $0.55 \times 0.32 \mathrm{~m}(\mathrm{~L} \times \mathrm{W} \times \mathrm{H})$. The full structure has 3 rectangular bases, each one for a specific objective. The bottom one, also the largest, serves as a structural/support base and is the one to be attached on the receiving platform. Along this, another structure slides to extend the cradle horizontally outside the volume. Between these exists another structure that gives the cradle rotational motion, enabling tilting. In Fig. 1 are presented the different configurations mentioned.

Given that a great concern of the authors is modularity of the system, the diameter restriction of the vehicles to be carried had to be rethought. So, the cradle was designed to be changeable, being only necessary to change this to transport vehicles of different diameter dimensions.

\section{B. Actuation}

Actuation enables the frames to move, using three $12 \mathrm{~V}$

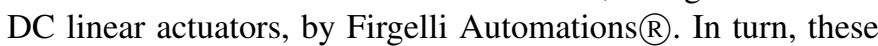



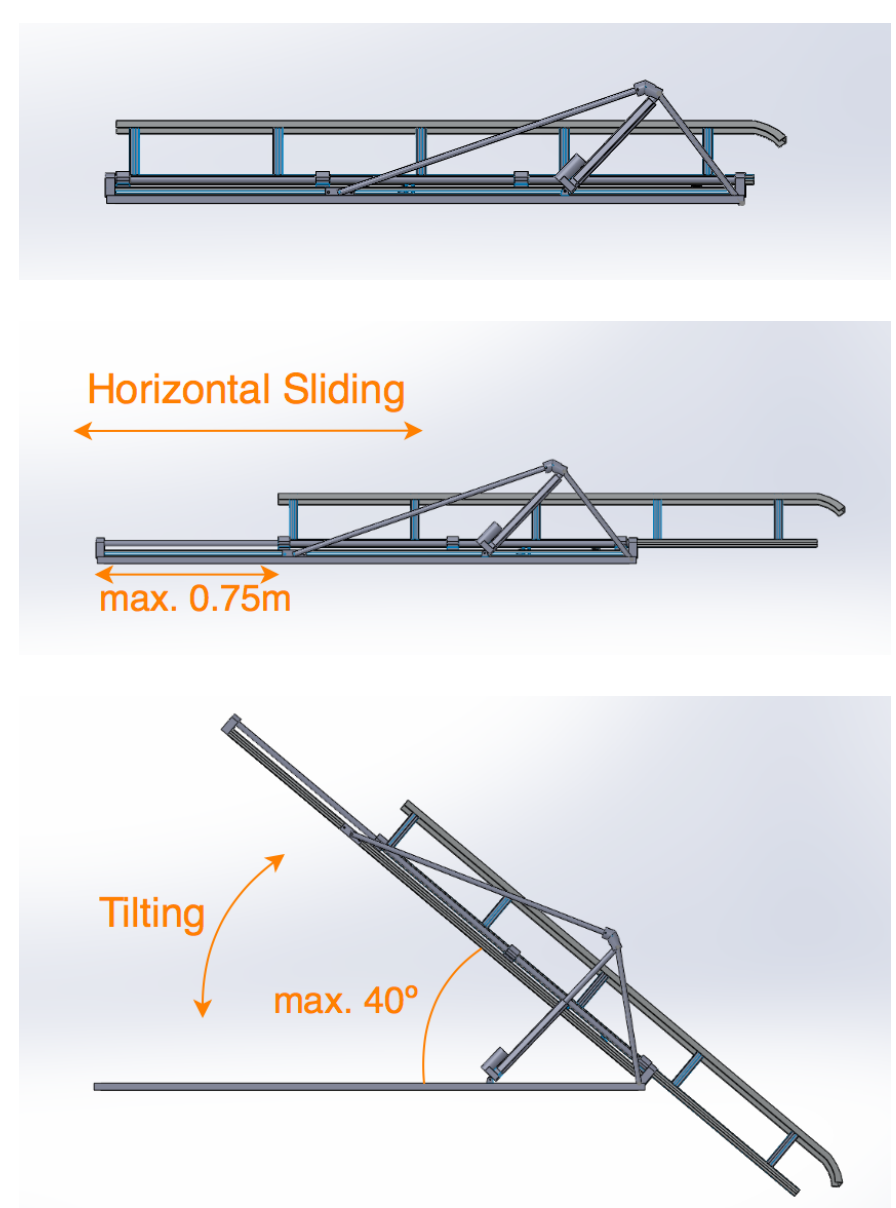

Fig. 1: Multiple Mechanical Positions

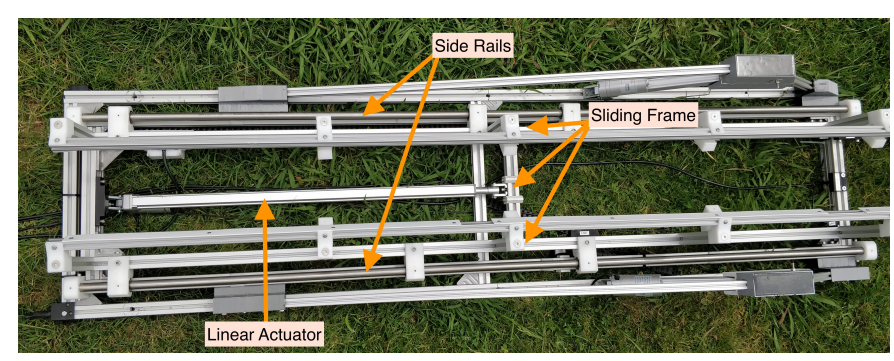

Fig. 2: Horizontal Movement Actuation

are actuating linear segments that provide horizontal and tilt movements to the frame where the vehicle stays. These actuators gives the system ability to perform the launching and recovering actions. Also, a DC motor is used to rotate a drum capable of winding and unwinding a cable, very similar to a winch.

For horizontal movement, there is used one FA-240-12-30"$\mathrm{P}$ linear actuator, moving the cradle up to $0.75 \mathrm{~m}$. The top frame slides through two side rails as can be seen in Fig.2.

For tilting, two FA-400-12-12"-P linear actuators are used, one on each side of the cradle to guarantee stability, to enable tilting movement. To perform this operation a structure

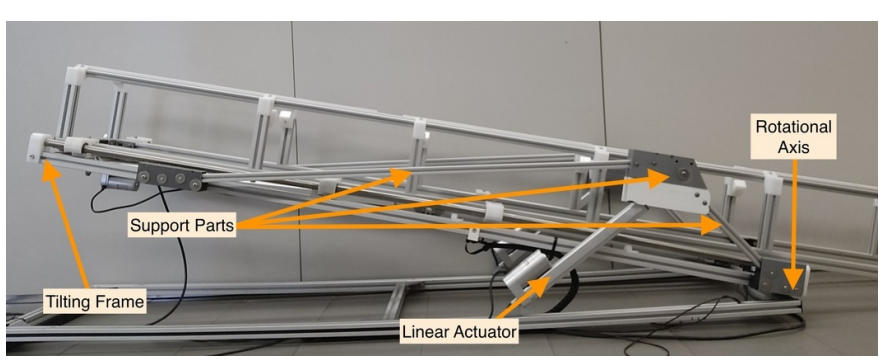

Fig. 3: Vertical Movement Actuation

consisting of 2 support bars and a joint is used, arranged triangularly, where one of the vertexes is the axis of fixed rotation. Then, the actuator is attached on one side to the fixed frame on the platform and the other to the joint, pushing the central vertex of the triangle. Since the remaining vertex is not fixed, this will tilt the frame. The structure is presented in Fig.3.

The maximum angle that the cradle tilts is $40^{\circ}$. Fig. 1 illustrates the possible movements, as well as maximum positions of the moving cradle.

An important note, before being presented in the next section, is that the used linear actuators have incorporated limit switches, stopping by themselves when reaching the end of their course. However, to create redundancy, additional limit switch sensors were used to stop the actuators before they reach the maximum limit.

\section{Sensing}

For remote monitoring and supervision, the addition of sensors to the mechanical structure is required to monitor a moving platform. In this specific case, sensors to monitor movement (horizontal and vertical), as well as limit switches, were used. Since environmental conditions may vary, optical sensors were excluded from these choices. Therefore, the following were used:

- Linear Displacement Sensor - RLS RMK4 is a magnetic angular position encoder for $360^{\circ}$, with 12-bit resolution and capable of presenting absolute and relative positions. This sensor is based on the hall effect and measures angular displacement. To obtain linear displacement from it, a rack and pinion mechanism was designed and built. A magnet has been placed in the center of the gear and, concentric with it, the hall sensor was potted in epoxy. The used rack was fixed side by side with the gear, allowing that, when the cradle moves, the gear rotates, as shown in Fig.4a. The rack and the pinion have been designed each with $10 \mathrm{~mm}$ of height, in order to accommodate some small misalignments during operation;

- Limit Switches - These are used to create safety redundancy, as mentioned previously, and stop the actuators at designated points. Magnetic sensors were used, since these are tolerant to displacement errors, do not require power to operate and can be potted in epoxy. So, ABUS MK1010B are used to verify and limit the horizontal 


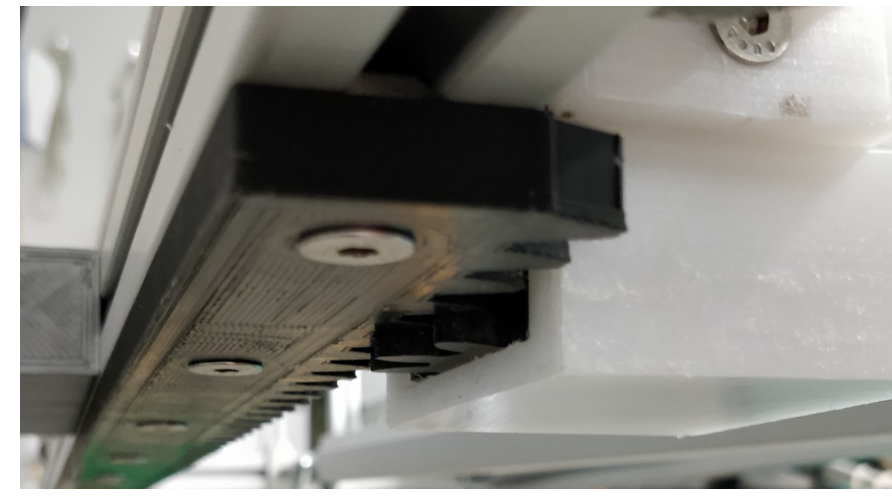

(a) Rack and Pinion Mechanism

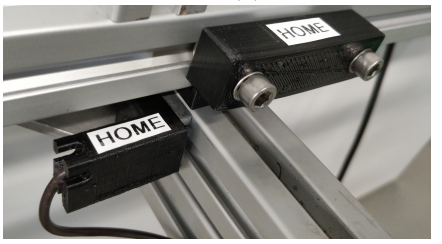

(b) Magnetic Limit Switch

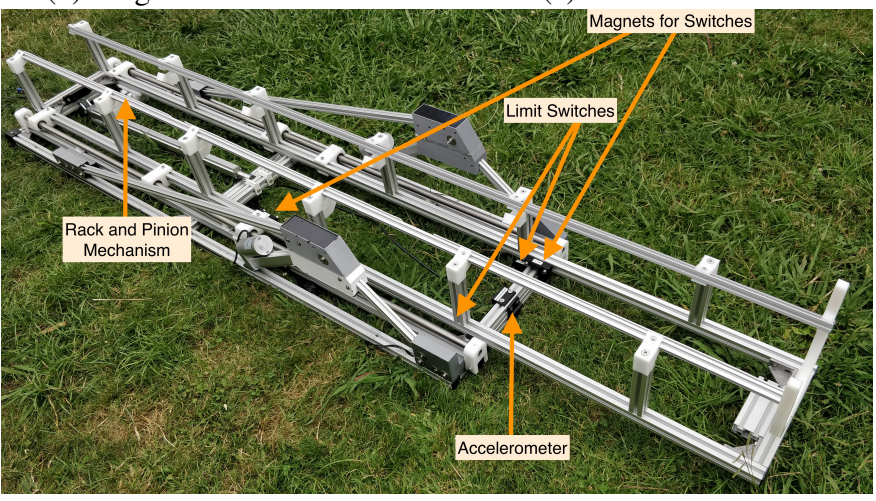

(d) Components Location on the System

Fig. 4: Sensors and their Location on the system

cradle and winch movements, together with Neodyium Iron Boron strong magnets. This way, the sensors accommodate shaking in the ALARS structure. To receive home and end sensors, identical structures were designed and placed in opposite sides of the cradle. An example of these is presented in figure $4 \mathrm{~b}$;

- Tilting Angular Sensor - Adafruit LIS3DH is a 3-DOF accelerometer breakout board used to provide information about the cradle's inclination. This accelerometer has a resolution of 10-bit, selectable scaling, data rate and low power mode. The board was also potted in epoxy on a specific designed shell to enable waterproofness, presented in figure 4c. Since only 1 DOF is required, this is the only information passed to the supervisor.

\section{Gateway Box}

One of the defined requirements in section III, was that the system should work with any host platform. In order to be fulfilled, it was defined that, from the host, only a network connection and a power source would be used. This must

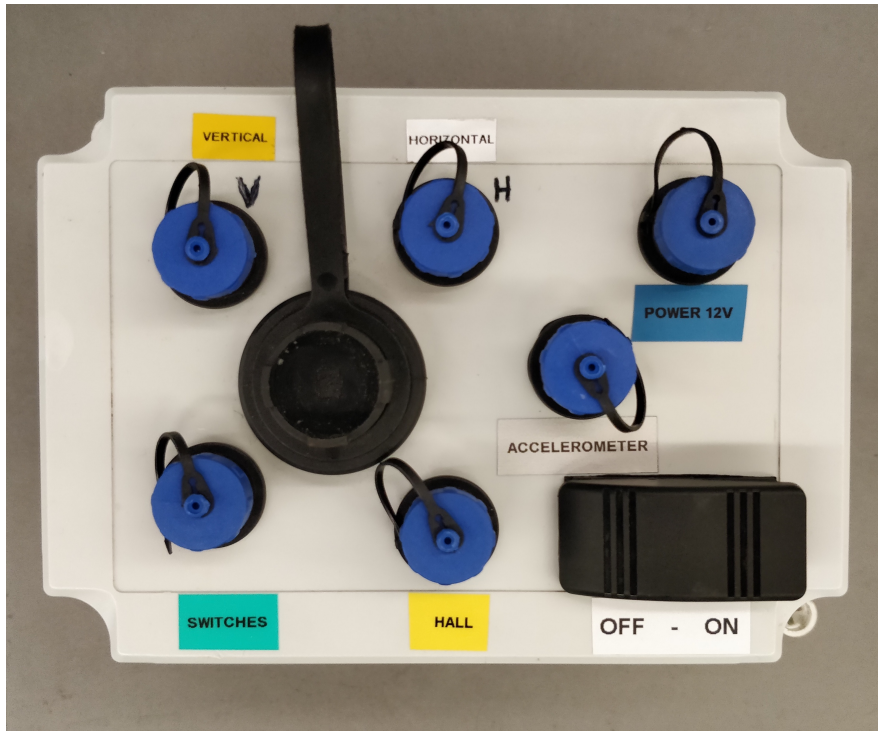

Fig. 5: ALARS Gateway Box (connector view)

provide a Service Set Identifier (SSID) that allows the box to connect to or, alternatively, an RJ45 Ethernet connection. Moreover, the network may allow Secure Shell (SSH), so it can be possible to remotely control the system.

So, with these ideas in mind, a gateway box was constructed to perform the "bridge" function between the host platform and the system (Fig.5). The watertight box is composed by the following components, connected as illustrated by Fig.6:

- Single Board Computer - this acts as central unit, has a Linux-based operating system and manages the whole system, accepting input commands and generating actions. To execute this function, a Raspberry Pi (RPi) board was chosen, more specifically the $3 \mathrm{~B}+$ model, since it supports both $2.4 \mathrm{GHz}$ and $5 \mathrm{GHz}$ Wi-Fi networks;

- DC-DC Voltage Regulator - although the power for the actuators come directly from the external power source, the RPi must be powered at 5V DC. For this, a DCDC Adjustable Voltage Regulator was used - Pololu S18V20ALV;

- Dual Motor Controllers - given that the motor group$\mathrm{s}$ may have to be actuated independently, dual motor controllers - Pololu TReX DMC01 (linear actuators) and TReX Jr DMC02 (winch) - were chosen to accomplish the objectives;

- $I^{2} \mathrm{CHub}$ - to facilitate the cable distribution and only access the generic RPi $\mathrm{I}^{2} \mathrm{C}$ bus, a hub was designed to aggregate all and route only one set of cables to this bus.

\section{SOFTWARE}

As previously stated, there should be created conditions for remote monitoring of the ongoing operation, as well as intervention. So, there should be created an interface that enables remotely controlled mode. It was chosen to create this interface through command line, considering regular mission 


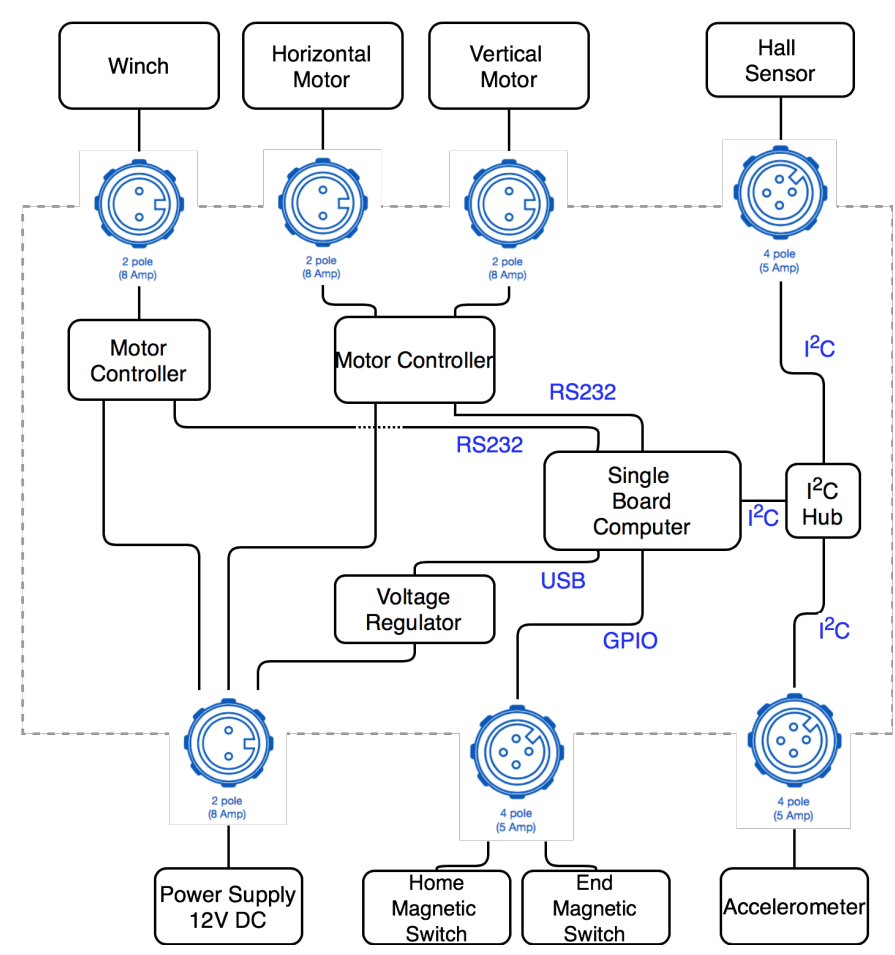

Fig. 6: ALARS Gateway Box Connections

conditions in field and since the processing time with SSH connection is very low.

As mentioned, the processing unit is a Raspberry $\mathrm{Pi} 3 \mathrm{~B}+$, with a Linux-based distribution. The programming language used to implement the scripts was $\mathrm{C}++$ and the drivers for each sensor and actuator were developed by the authors. The chosen policy was to produce software capable of operating without relying on any library external to the general Linux environment. Therefore, independent drivers were created for both sensors and actuators, making them usable in any Linuxbased operating system. Since two of the sensors use an $\mathrm{I}^{2} \mathrm{C}$ protocol and another uses information from the GPIO, independent software was also created to manage this.

\section{A. Actions}

First, a set of low level functions were created, allowing to perform initializations or calibrations. In a mission context, as an example, the accelerometer must need to calibrated in the operation site.

Then, a set of structured commands were defined. Examples include "Horizontal Forward", "Vertical Forward", "Winch Forward" and "Stop". Furthermore, the developed procedures use these commands to perform the actuations.

\section{B. Procedures}

Procedures are a set of controlled events to achieve a complete goal, as "Launch", "Recover" and "Retract".

For the "Launch" procedure, cradle is typically in the initial (Home) position. Then, the cradle will travel horizontally approximately $750 \mathrm{~mm}$ outside the fixed frame, always maintaining relative roll, pitch and yaw angles, until the end

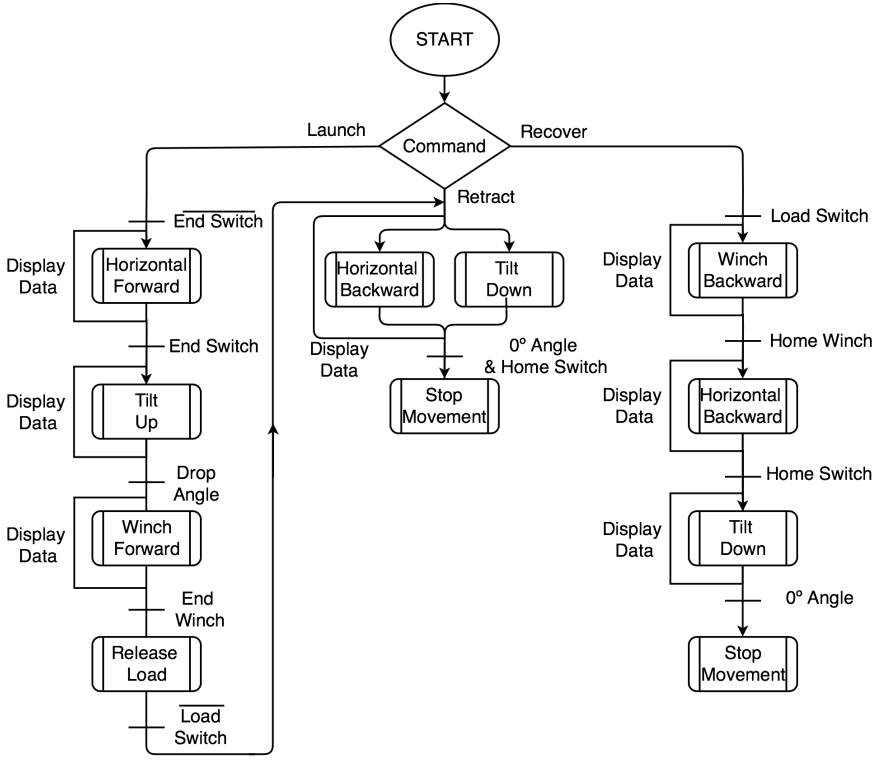

Fig. 7: Launch and Recovery Software Procedures

switch is activated. After, it tilts to an angle of approximately 25 degrees, where around $0.2 \mathrm{~m}$ of the cradle's bottom is underwater. At this point, the winch makes the AUV to slide down until the end winch switch is triggered, while it is still attached. The restraining system is then disabled, releasing the AUV.

When the AUV leaves the cradle, ALARS is triggered to "Retract" to the initial position. For this, both horizontal and vertical actuators start to run backwards, for the procedure to be quicker. These actions stop when the cradle arrives at the (Home) position, with $0^{\circ}$ angle.

To "Recover" the AUV, it must be at the end switch, with $25^{\circ}$ angle. Then, the winch will pull the AUV to the top of the cradle, until the winch home switch is triggered. Finally, in turn, the cradle is pulled horizontally until the Home switch and then is tilted down until reaching $0^{\circ}$ angle and then, stopping the movement.

All this information is aggregated in the software architecture diagram (Fig.7).

\section{EXPERIMENTAL RESULTS}

The ALARS was tested on December 20th, 2017, in the Leixões Harbor, Matosinhos, Portugal, during the XPRIZE demonstrations of the PISCES team. The host platform was the ROAZ II ASV [9] and the transported vehicle was the DART AUV [10]. The "Launch" procedure was performed, triggered remotely, without any further human intervention.

Through the logs produced by the system during operation, the whole launch procedure took around 120 seconds. For horizontal sliding and tilting, the system took around 80 and 40 seconds, respectively. The power consumption of each step, with the vehicle on top of ALARS was $30 \mathrm{~W}$.

The whole experimental can be checked in Fig. 8, that contains a photograph sequence took that day. 

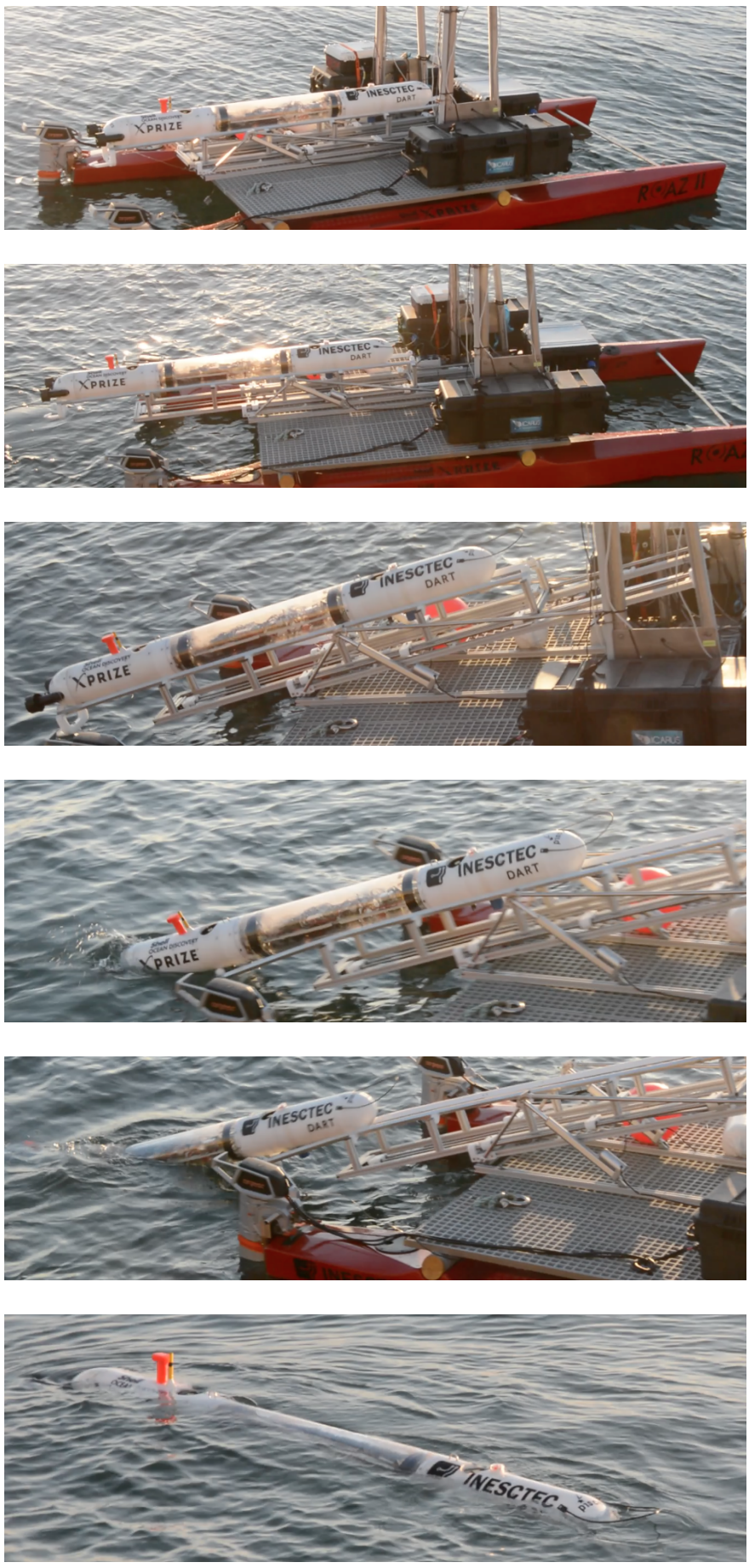

Fig. 8: Experimental Launch Procedure

\section{CONCLusions And Future Work}

As described along the paper, the developed ALARS is ready for further field tests. It was implemented bidirectional automated actuation, allowing for forward and backward movements without the need for continuous remote operation. The integrated sensors allowed not only remote monitoring and operation, but goes further and allows the system to launch autonomously. These features have already been tested and validated in the experimental results presented in the previous section. However, the vehicle was launched only due to the tilting mechanism.

In the next opportunity to test the system, the winch will be used to limit the sliding speed, enabling the flooded sections to fill slowly, as well as to perform the vehicle's recovery back to the home position.

\section{ACKNOWLEDGMENTS}

This work is financed by the ERDF - European Regional Development Fund through the Operational Programme for Competitiveness and Internationalisation - COMPETE 2020 Programme within project "POCI-01-0145-FEDER-006961", and by National Funds through the Portuguese funding agency, FCT - Fundação para a Ciência e a Tecnologia as part of project "UID/EEA/50014/2013".

\section{REFERENCES}

[1] N. A. Cruz, N. Abreu, J. Almeida, R. Almeida, J. Alves, A. Dias, B. Ferreira, H. Ferreira, C. Gonçalves, A. Martins et al., "Cooperative deep water seafloor mapping with heterogeneous robotic platforms," in OCEANS'17 MTS/IEEE Anchorage. IEEE, 2017, pp. 1-7.

[2] C. L. Kaiser, D. R. Yoerger, J. C. Kinsey, S. Kelley, A. Billings, J. Fujii, S. Suman, M. Jakuba, Z. Berkowitz, C. R. German et al., "The design and 200 day per year operation of the Autonomous Underwater Vehicle Sentry," in Autonomous Underwater Vehicles (AUV), 2016 IEEE/OES. IEEE, 2016, pp. 251-260.

[3] "AUV Sentry Launch and Recovery Tutorial Video," http://www.whoi. edu/page.do?pid=38095\&tid=7842\&cid=123093, Last Accessed: 2018 07-16.

[4] D. Thompson, D. Caress, H. Thomas, and D. Conlin, "MBARI mapping AUV operations in the gulf of California 2015," in OCEANS'15 MTS/IEEE Washington. IEEE, 2015, pp. 1-7.

[5] E. Hayashi, H. Kimura, C. Tam, J. Ferguson, J.-M. Laframboise, G. Miller, C. Kaminski, and A. Johnson, "Customizing an Autonomous Underwater Vehicle and developing a launch and recovery system," in Underwater Technology Symposium (UT), 2013 IEEE International. IEEE, 2013, pp. 1-7.

[6] E. I. Sarda and M. R. Dhanak, "A USV-Based automated launch and recovery system for AUVs," IEEE Journal of Oceanic Engineering, vol. 42, no. 1, pp. 37-55, 2017.

[7] J. C. Piskura, M. Purcell, R. Stokey, T. Austin, D. Tebo, R. Christensen, and F. Jaffre, "Development of a robust Line Capture, Line Recovery (LCLR) technology for autonomous docking of AUVs," in OCEANS'16 MTS/IEEE Monterey. IEEE, 2016, pp. 1-5.

[8] N. A. Cruz and A. C. Matos, "The MARES AUV, a modular autonomous robot for environment sampling," in OCEANS'08 MTS/IEEE Quebec. IEEE, 2008, pp. 1-6.

[9] A. Martins, H. Ferreira, C. Almeida, H. Silva, J. M. Almeida, and E. Silva, "ROAZ and ROAZ II autonomous surface vehicle design and implementation," in International Lifesaving Congress 2007, 2007.

[10] N. A. Cruz, A. C. Matos, R. M. Almeida, and B. M. Ferreira, "DART - a portable deep water hovering AUV," in OCEANS-Anchorage, 2017. IEEE, 2017, pp. 1-6. 